\section{A case of esophageal perforation: Clinical and diagnostic management in emergency medicine}

\author{
Marco Di Serafino, ${ }^{1}$ Maurizio Martorano, ${ }^{2}$ \\ Pamela Delmonaco, ${ }^{3}$ Chiara Gullotto, ${ }^{4}$ \\ Andrea Baglioni, ${ }^{5}$ Leonardo Martinelli, ${ }^{4}$ \\ Riccardo Savastano, ${ }^{6}$ Enrico Scarano, ${ }^{1}$ \\ Francesco Lisanti ${ }^{2}$ \\ 'Radiology Department, San Carlo \\ Hospital, Potenza; 'Intensive Care Unit, \\ San Carlo Hospital, Potenza; ${ }^{3}$ Emergency \\ Department, San Carlo Hospital, Potenza; \\ ${ }^{4}$ Emergency Department, Cisanello \\ Hospital, Pisa; ${ }^{5}$ Careggi University \\ Hospital, Florence; 'San Giovanni di Dio \\ e Ruggi d'Aragona University Hospital, \\ Salerno, Italy
}

\begin{abstract}
Esophageal perforation is a welldefined and severe clinical condition. The associated mortality rates range between $5 \%$ and $40 \%$ and are worsened by delayed diagnosis. Rapid diagnosis and therapy provide the best chance for survival; however, a delay in diagnosis is common, resulting in substantial morbidity and mortality. There are several aetiologies of esophagus perforation. Most esophageal ruptures are secondary to medical instrumentation. Other causes are Boerhaave syndrome, toxic ingestions and radiation, foreign body ingestion, penetrating trauma, and, rarely, blunt chest trauma. We reported the clinical management and the diagnostic work-up case of esophagus perforation due to the foreign body ingestion.
\end{abstract}

\section{Introduction}

Esophageal perforation is a serious injury of the gastrointestinal tract. The associated mortality rates range between 5\% and $40 \%{ }^{1}$ and are worsened by delayed diagnosis. Rapid diagnosis and therapy provide the best chance for survival; however, a delay in diagnosis is common, resulting in substantial morbidity and mortality. There are several etiologies of esophagus perforation. Most esophageal ruptures are secondary to medical instrumentation. Other causes are Boerhaave syndrome, toxic ingestions and radiation, foreign body ingestion, penetrating trauma, and, rarely, blunt chest trauma. Esophageal perforation often exhibits large clinical variability in its pres- entation (chest/neck pain, dysphagia, hematemesis, pleural effusion, pneumomediastinum, subcutaneous emphysema, empyema, peritonitis and sepsis can frequently be found), representing therefore a diagnostic and therapeutic challenge because of the rarity of the condition and its variability in the presentation.

\section{Case Report}

A middle-aged disabled man, cared for in a psychiatric hospital, came to our attention at the Emergency-Urgency Department for a severe dyspnea and vomiting of blood. It was reported that he had ingested a sandwich with voracity before the onset of suffocating symptoms with severe dyspnea, coughs and vomiting contractions. On examination, he was clutching his chest in pain and spitting blood with mild epigastric tenderness. He had tachycardia and tachypnea but no hypotension or fever. His hematocrit was also normal. He wore a complete upper denture. In the first examination, his vital signs were stable but he was found to have neck and thorax crepitus. He was subjected to a chest X-ray that showed widespread subcutaneous emphysema with thoracic-abdominal and neck extension, pneumomediastinum and a small fence of bilateral penumothorax (Figure 1). Because of the ingravescent subcutaneous cervical-thorax emphysema and dyspnea the patient required endotracheal intubation for the appearance of hemodynamic instability, which has given priority to mechanical ventilation, procrastinating the bilateral pneumothorax drainage. A computed tomography (CT) was also performed and it demonstrated an upper third lesion of the esophagus (Figure 2) with an extraluminal massive air spread characterized by cervical and thoracic-abdominal subcutaneous emphysema, pneumomediastinum and bilateral penumothorax (Figure 2). At the same time, CT showed a sharp foreign body, like a cutting blade of about $4 \mathrm{~cm}$ in diameter, in the gastric lumen (Figure 2): this find suggested an esophageal lesion from a foreign body. According to the critical health condition of the patient he was admitted to our Intensive Care Unit. A right side PICC (peripherally inserted central catheter) was correctly inserted and broad spectrum antibiotic therapy (Clindamycin $450 \mathrm{mg}$ EV every 6 hours and Ceftriaxone $2 \mathrm{~g}$ once a day) was started. A nasogastric tube was also inserted under laryngoscopy vision for enteral feeding. To a stabilized health patient's condition was planned an urgent surgery-esophagocopy. A pre-operative esophagography with diatrizoate meglumine (Gastrografin) was also
Correspondence: Marco Di Serafino, Radiology Department, San Carlo Hospital, via Potito Petrone 85100 , Potenza, Italy. Tel.: +39.0971613315 - Fax: +39.0971613315 E-mail: marcodiserafino@hotmail.it

Key words: Esophageal perforation, pneumomediastinum, pneumothorax, subcutaneous emphysema.

Contributions: MDS, CG made substantial contributions to conception, design and in drafting the article. $\mathrm{PD}, \mathrm{AB}, \mathrm{LM}$ participated in revising it critically for important intellectual content. MDS, ES interpreted the radiologic examination findings and the patient data regarding the differential diagnosis of the disease. RS made substantial contributions to acquisition, analysis and interpretation of data. MM, FL designed and reviewed the manuscript and gave final approval of the version to be submitted. All authors read and approved the final manuscript.

Conflict of interest: the authors declare no potential conflict of interest.

Received for publication: 11 June 2017. Revision received: 24 October 2017.

Accepted for publication: 26 October 2017.

This work is licensed under a Creative Commons Attribution 4.0 License (by-nc 4.0).

CC Copyright M. Di Serafino et al., 2017 Licensee PAGEPress, Italy

Emergency Care Journal 2017; 13:6848

doi:10.4081/ecj.2017.6848

performed to better identify the site of oesophageal lesion. This latter showed a large spread of the hydrous fluid contrast medium at the level on the left side of the esophageal cranial portion (Figure 3). An esophageal endoscopy confirmed the esophageal perforation (Figure 3) evaluating its extension and the gastric position of the foreign body. Because of the size of the lesion of about $5 \mathrm{~cm}$ in diameter and the involvement of the upper esophagus was opted for a surgical repair and a laparoscopic removal of the gastric foreign body considered the high risk of its endoscopic removal. Direct suture of perforation was obtained by laparoscopic approach and protection of the suture was realized with reinforcement flap obtained with gastric muscle portion. After two days, a neck and chest radiogram revealed progressive reabsorption of air and an esophagography with Gastrografin showed no further spills of water-soluble contrast medium neither at cranial esophagus nor at the post-laparoscopic gastric area of surgery. The patient was successfully extubated and subsequent- 
ly dismissed to the hospital care as soon as there was an improvement in his physical condition and he was entrusted with social services.

\section{Discussion}

Esophageal perforation is a serious injury of the gastrointestinal tract. The associated mortality rates range between 5\% and $40 \%{ }^{1}$ and are worsened by delayed diagnosis. If the treatment is started after 24 hours of the injury, the mortality rate can increase to $50 \% .^{1}$ Since esophageal perforation was originally described more than 50 years ago, the diagnosis has been challenging, its management is controversial and mortality is still high. ${ }^{2}$ The clinical course of esophageal perforation essentially depends on the location and the extent of the injury as well as the time elapsing between the perforation and the start of the treatment. ${ }^{3}$ Esophageal perforation may result from iatrogenic, penetrating or blunt trauma and foreign body ingestion too, as in our case. However, by far the commonest traumatic perforation of the esophagus results from iatrogenic action. The causes of iatrogenic esophageal perforation include endoscopic procedures, nasogastric tube insertion, difficult endotracheal intubation, percutaneous tracheostomy, surgery of the mediastinal organs including resection of lung cancer, operations on the cervical spine, thyroidectomy, and palliative intubation, stenting, or laser treatment of esophageal tumors. ${ }^{4}$ Foreign body ingestion, penetrating trauma, and corrosive injury are other causes of perforations. ${ }^{5}$ Owing to its protected position, the esophagus is only rarely perforated by penetrating wounds, though these include inadvertent damage during surgery in the neck and especially in the region of the esophageal

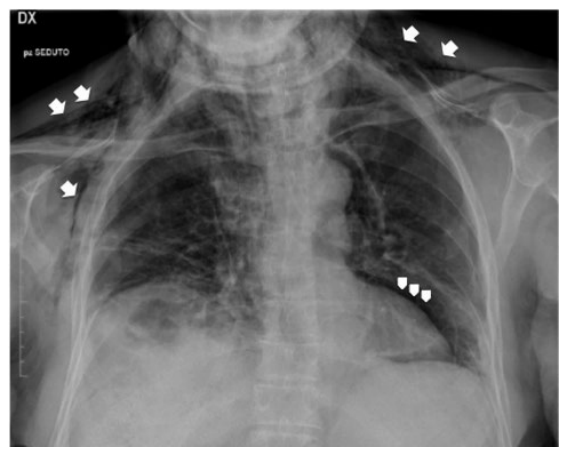

Figure 1. Antero-posterior chest radiograph shows subcutaneous emphysema (white arrows) and pneumomediastinum (white arrowheads). hiatus in the diaphragm. Instead, spontaneous rupture of the esophagus accounts in only $10-35 \%$ of all perforations of the esophageal wall ${ }^{2}$ and the rupture is usually longitudinal, from 2 to $6 \mathrm{~cm}$ long, and located at the left side of the lower third of the esophagus. ${ }^{6}$ The aetiology has been attributed to an anatomic weakness resulting from the reduced number and size of the longitudinal smooth muscle fibers, from the entrance of nerves and vessels in the wall, and from the lack of buttressing structures. ${ }^{6}$

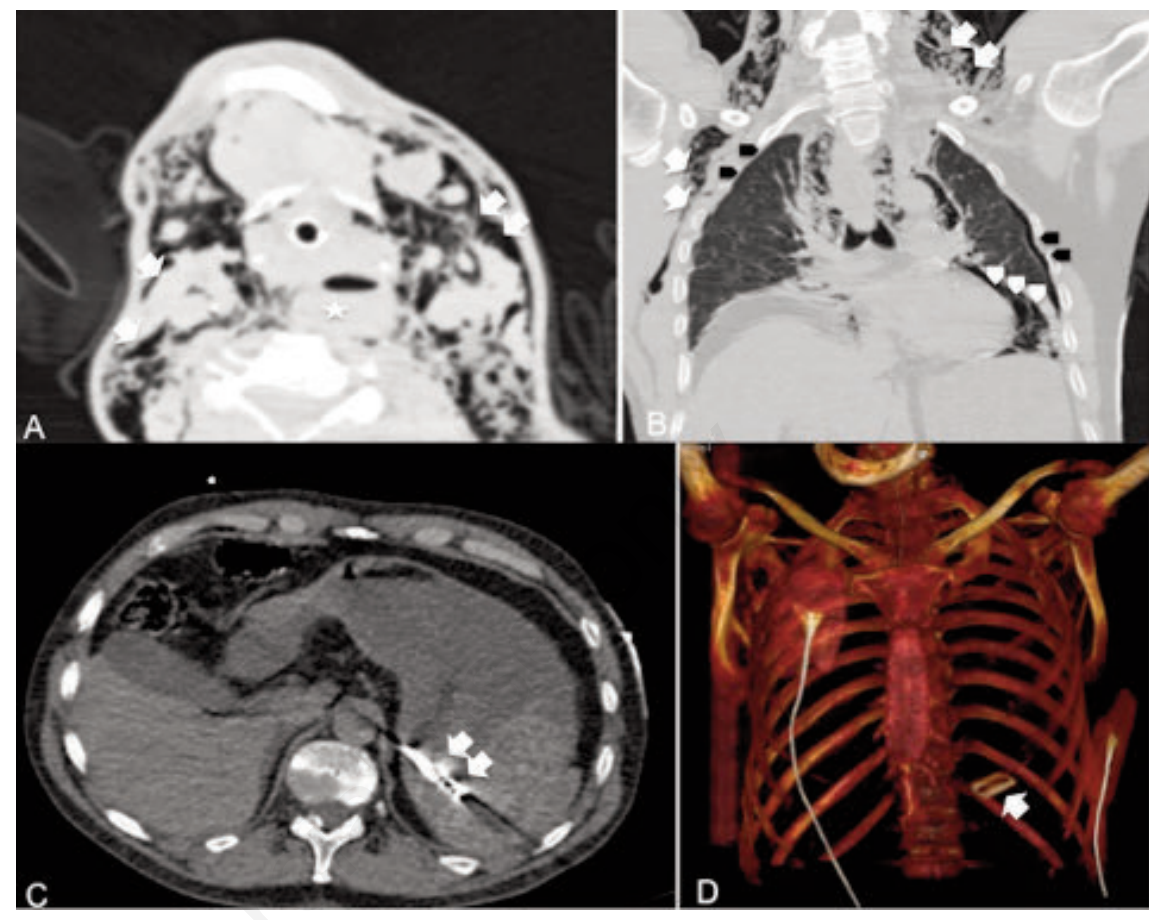

Figure 2. Non-enhanced CT scan axial (A), coronal (B) magnified images show the esophageal perforation (star) and an extra-luminal massive air spread characterized by cervical and thoracic-abdominal subcutaneous emphysema (white arrows), pneumomediastinum (white arrowheads) and bilateral penumothorax (black arrowheads). The nonenhanced CT scan axial (C) and 3D-Volume Rendering Coronal reconstruction (D) images show a sharp foreign body, like a cutting blade in the gastric lumen (white arrows).

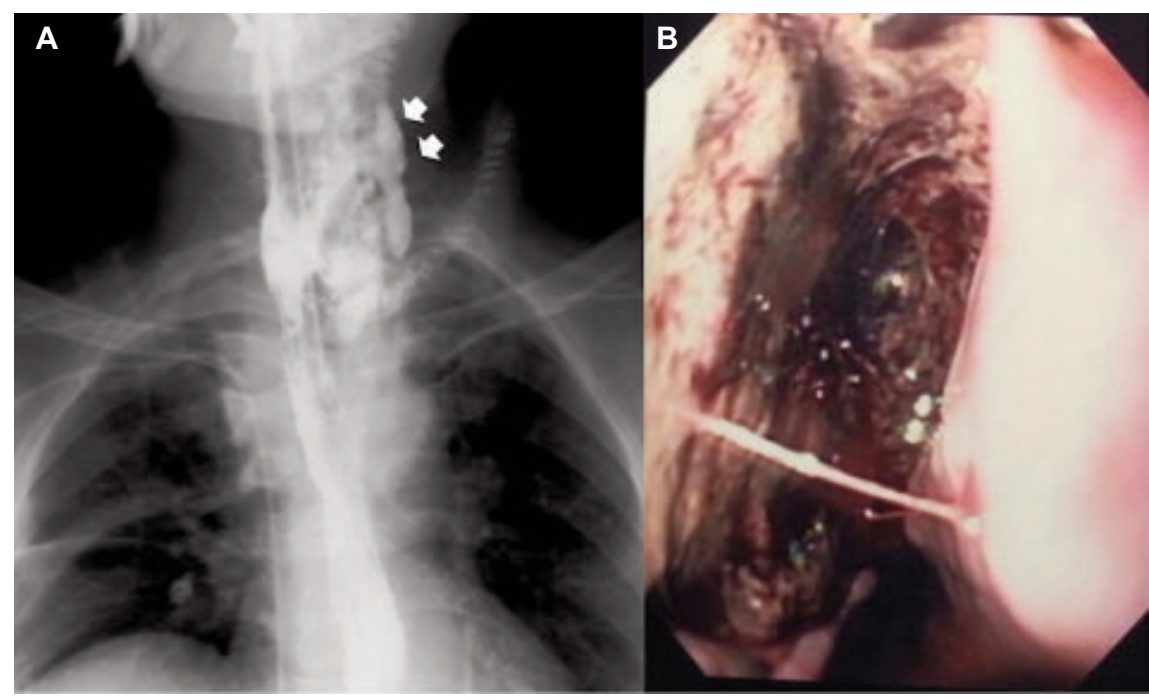

Figure 3. A contrast-swallow study (A) shows leakage of contrast agent at the level of the left side upper esophagus (white arrows) due to esophageal perforation confirmed by endoscopy view (B). 
As in our case, esophageal perforation is often secondary to foreign body ingestion, as foreign body ingestion is a common occurrence in children and in specific highrisk groups of adults, such as those with underlying esophageal disease, prisoners, the mentally retarded, and those with psychiatric illnesses. Although most foreign bodies pass through the gastrointestinal tract without difficulty, sharp, pointed, and elongated foreign bodies are associated with a greater risk of perforation, vascular penetration, and other complications. Foreign body ingestion is usually diagnosed based on a history of ingestion given by the patient or an observer. However, children and impaired adults may be unable to give an accurate history, and a high index of suspicion must be maintained in these groups; ${ }^{7}$ in our case, in fact, a disabled patient came to our observation with a choking syndrome without a certain history of ingestion of a sharp knife. The commonest sites of perforation are at the anatomical as well as pathological areas of narrowing. ${ }^{7}$ There are areas of anatomical esophageal narrowing, including the cervical esophagus, as in our case, aortic-arch, and gastro-esophageal junction which is more prone for foreign body impaction. ${ }^{8}$ The incidence of perforation caused by foreign bodies trapped in the esophagus is variable. Ingested foreign bodies are responsible for $80 \%$ of the cervical perforations and for $9-35 \%$ of all esophageal perforations. ${ }^{9}$ The perforation may be directly due to the action of sharp or piercing foreign bodies or to tissue necrosis following the pressure exerted by the foreign body at the site of impact. ${ }^{9}$ Perforations of the cervical esophagus are anyway infrequent severe situations, which due to the characteristics of the esophagus (topography, septic content and the continual movements associated with swallowing and breathing); perforations in this area may even be life threatening for patients. ${ }^{5}$ However, perforations of the cervical esophagus have a better prognosis than those involving other levels of the esophagus which are associated with a high degree of morbidity and mortality. ${ }^{10}$ The incidence of esophageal perforations appears to be increasing and the problems associated with recognition and treatment of this often-catastrophic occurrence remain a challenge. Perforated esophagus is an emergency, in fact it is the most serious and frequently the most rapidly lethal perforation of the gastro-intestinal tract, usually fatal if untreated. Contamination of the mediastinum and pleural cavity with corrosive fluids, food matter and bacteria lead to cardiorespiratory embarrassment, shock, major fluid losses and fulminating infection. With prompt, aggressive surgical treatment, survival can be expected in most cases. ${ }^{11}$ Clinical presentation may vary from paucisintomatic forms to severe forms often complicated by sepsis. Anyway, dysphagia and odynophagia are the usual symptoms of foreign body impaction in the esophagus. Respiratory symptoms due to compression of the adjacent trachea are also common in younger children and are occasionally the presenting symptom in adults. ${ }^{7}$ Classic clinical presentation, as usually described in literature referring to a particular type of esophageal perforation known as Boerhaave's syndrome, is of food or drink overindulgence with consequent vomiting followed by severe chest pain, dyspnea, mediastinal or subcutaneous emphysema (Meckler's triad), like in our case, and cardiovascular collapse. ${ }^{12}$ However, some reviews suggest that in most of the cases the presence of the entire complex of symptoms is rare, and therefore reliance on a classic presentation might be misleading, ${ }^{12}$ in fact, only in approximately $50 \%$ of the cases, the classic sequence of forceful vomiting, mild hematemesis, and sub-sternal chest pain was present. ${ }^{12}$ Unusual clinical features such as a change in voice, extreme swelling of face and neck, cold water polydipsia/thirst, pericarditis, pneumopericardium, pneumoperitonium, proptosis, low pleural fluid amylase should also be kept in mind while evaluating patients with suspicion of esophageal rupture. ${ }^{12}$ The most striking feature of this condition is the excruciating pain which is poorly relieved by narcotics. It usually presents as a pleuritic left-sided chest pain which may radiate to the sub-sternal area, epigastrium or back. Swallowing exaggerates the pain and it may cause coughing if there is a pleural tear. Sometimes the patient may collapse and dyspnea usually develops after the onset of pain because of splinting, hydropneumothorax or a tension pneumothorax and pain itself. ${ }^{13}$ It must be remembered that a hydropneumothorax or a tension pneumothorax may be contributing significantly to the patient's respiratory impairment. ${ }^{14}$ Another clinical presentation is the presence of subcutaneous emphysema. Although very helpful, it is rarely present initially, either because perforation has occurred directly into the pleural cavity or because there was no time for it to develop radiographically. ${ }^{12,14}$ Another clinical presentation is that of the spontaneous pneumothorax with all the characteristic signs such as tracheal deviation, hyperresonance, loss of retrosternal dullness, and decreased breath sounds, anyway physical examination of the chest may be completely normal in the early stages. ${ }^{14}$ Perforation can be complicated by inflammatory phenomena. As the inflammatory process subsequently starts in the mediastinum and pleural cavities, most patients become febrile, accompanied by signs of septicaemia and haemodynamic instability; ${ }^{2}$ they may often complain of extreme thirst, as is seen in the hypovolemic states and have a tachycardia due to a combination of hypovolemia and possible bacteremia. The blood pressure may be decreased and they may be mildly febrile in the early stages or vital signs may be within normal limits in the first crucial twelve hours particularly prior to rupture of the mediastinal pleura. ${ }^{14}$ Usually, diagnosis requires radiologic and endoscopic examination. Various types of imaging can be used as diagnostic tools. A chest radiograph is a good preliminary test, although it is quite often normal. ${ }^{15}$ The most common findings with chest radiograph are pleural effusions and pneumothorax with incidences of $91 \%$ and $80 \%$, respectively. Pneumothorax is usually associated with pleural effusion and is commonly found unilateral, but can be bilateral in a few cases as in our case. ${ }^{16}$ Subcutaneous emphysema in the soft tissue of the neck or chest wall and the mediastinal air is seen in $66 \%$ of patients. ${ }^{16}$ Mediastinal emphysema takes at least one hour to develop and chest radiograph remains normal in $10-12 \%$ of patients. ${ }^{15}$ Therefore, if there is a suspect of esophageal rupture, whether or not mediastinal emphysema is detected, it is advisable to do a contrast study of the esophagus. ${ }^{15}$ Contrast esophagography is the standard technique for diagnosing esophageal perforation and can be performed with water-soluble contrast material (e.g. Gastrographin). In most cases, the site of the perforation is readily detected. Nevertheless, false-negative findings have been reported in up to $10 \%$ of patients. ${ }^{16}$ In patients in which esophageal perforation is clinically suspected and contrast studies are negative, computed tomography (CT) can be a good and useful diagnostic adjunct. ${ }^{17}$ CT is ideally suited for defining the extent of extra-luminal air and fluid. Moreover, thoracic CT can be complementary to a positive oral contrast study to localize collections of fluid for surgical drainage. ${ }^{17} \mathrm{CT}$ findings suggestive of esophageal perforation are: air in the soft tissues of mediastinum surrounding the esophagus; abscess cavities adjacent to the esophagus in either the pleural space or the mediastinum; demonstration of an actual communication between the air-filled esophagus and an adjacent mediastinal or paramediastinal airfluid collection. ${ }^{17}$ The abnormalities seen on CT scans may be the first imaging findings to suggest the diagnosis, as is true in $33 \%$ of 
patients. The efficacy of CT results from its use as a survey technique in confusing or complicated clinical situations that may result from esophageal perforation. Extraluminal air is the most useful CT finding: it occurs in $92 \%$ of cases. Additional CT findings such as esophageal thickening may allow further characterization of the underlying process. Mediastinal, cervical, pleural, or penicardial fluid is usually present but is a less specific finding. ${ }^{17} \mathrm{CT}$ scans are also useful in follow-up after initiation of therapy and in the evaluation of patients who fail to improve despite either operative or inoperative management. ${ }^{17}$ If the esophageal perforation is caused by a foreign body, the preferred method of removal is extracted with the flexible endoscope but in our case the non-endoscopic surgical removal was preferred both for the gastric localization and its sharp morphology. The management of the syndrome remains controversial since treatment can be surgical or non-surgical, and indications vary according to the functional state of the esophagus, the presence of associated lesions and the habits of the medical teams. ${ }^{18}$ Much attention has focused on therapeutic options in esophageal perforation. Clinically unstable patients with esophageal perforation require rapid resuscitation and treatment. Broadspectrum intravenous antibiotics should be initiated early and patients should receive nothing by mouth, and a nasogastric intubation should be considered to eliminate oral and gastric secretions. Early surgical consultation is warranted. ${ }^{19}$ Preferred surgical technique if lesion is located in the lower third of the esophagus is a left thoracotomy in the seventh or eighth intercostal space, although the transabdominal route can be used. ${ }^{19}$ Lesions affecting middle/upper thoracic esophagus, instead, are operated through right thoracotomy, while cervical esophagus lesions are treated with left neck incision. ${ }^{20}$

A suite of endoscopic modalities can also be used in treatment of esophageal perforation. Endotherapy techniques are a recent addition to the suite of non-surgical and minimally invasive strategies to manage patients with esophageal perforations. Endoscopic approaches not only seek to minimize invasive surgery and trauma to patients but they also present challenges due to the acuity of presentation and variability of the underlying pathologies. Endotherapies aim to reproduce surgical strategies of tissue closure, diversion of enteric stream (stenting), drainage, and stricture management. Many of these techniques can be used in combination with others, and if a patient fails to obtain a successful outcome with their initial management then other alternatives can be used. Endoscopic options that can be employed include self-expanding metal stents (SEMS), pneumatic dilation, pigtail stents for internal drainage, tissue apposition using clips - through-the-scope clips (TTSC) or over-the-scope clips (OTSC) fistula plugs or glue. Fully covered SEMS (FCSEMS) are generally sutured in place surgically when they are deployed, and if surgery is not planned, self-retaining partially covered SEMS (PCSEMS) are used. Patients with clean or instrumental perforations are treated by direct closure with clips if defects are small or by endoscopic stent placement without external drainage if perforation is large or associated with luminal stenosis. $^{21}$

Although surgery remains the mainstay of treatment, intensive non-operative management is also possible in carefully selected patients with spontaneous esophageal rupture.

Success depends on a multidisciplinary approach with continuous reassessment and a low threshold for intervention. ${ }^{22}$

The prognosis of cervical esophageal injuries depends on many factors such as associated injuries, shock on admission, mechanism of injury and timing of operation: in our case prognosis was good because of the early detection and treatment of the lesion.

\section{Conclusions}

Foreign body ingestion in adults is not uncommon in the emergency department. Careful history taking is a key element of correct diagnosis and physical examination should be followed by the use of radiographic approach. Emergency should understand the variability of presentation of foreign bodies in the esophagus and make a proper physicians decision regarding treatment.

\section{References}

1. Henderson E, Echavé V, Lalancette M, Langlois G. Esophageal perforation in closed neck trauma. Can J Surg 2007;50:E5-6.

2. Brinster CJ, Singhal S, Lee L, et al. Evolving options in the management of esophageal perforation. Ann Thorac Surg 2004;77:1475-83.

3. Fernandez FF, Richter A, Freudenberg $\mathrm{S}$, et al. Treatment of endoscopic esophageal perforation. Surg Endosc 1999;13:962-6.

4. Gupta NM, Kaman L. Personal man- agement of 57 consecutive patients with esophageal perforation. Am J Surg 2004;187:58-63.

5. Hinojar AG, Díaz Díaz MA, Pun YW, Hinojar AA. Management of hypopharyngeal and cervical esophageal perforations. Auris Nasus Larynx 2003;30:175-82.

6. Korn O, Oñate JC, López R. Anatomy of the Boerhaave syndrome. Surgery 2007;141;222-8.

7. Sng KK, Koh JHA, Ngian-Chye T, et al. An Eastern perspective on oesophageal perforation: as high incidence of ingested bones. Anz J Surg 2008;78:573-8.

8. Alavinejad P, Ahmadi B, Akhavan K et al. Esophageal perforation with fish bone: a case report. Int Inv J Med Med Sci 2015;2:62-5.

9. Chirica M, Champault A, Dray X, et al. Esophageal perforations. J Visc Surg 2010;147:117-28.

10. Ryom P, Ravn JB, Penninga L, et al. Aetiology, treatment and mortality after esophageal perforation in Denmark. Dan Med Bull 2011;58:A4267.

11. Nealon TFJr, Templeton JW III, Cuddy VD, Gibbon JHJr. Instrumental perforations of the esophagus. J Thorac Cardiov Surg 1961;41:75-104.

12. Walker WS, Cameron EW, Walbaum PR. Diagnosis and management of spontaneous transmural rupture of the oesophagus (Boerhaave's syndrome). Br J Surg 1985;72:204-7.

13. Shenfine J, Griffin SM. Esophageal emergencies. In: Griffin SM, Raimes SA, Shenfine J, eds. Oesophagogastric surgery: a companion to specialist surgical practice. 3rd ed. Philadelphia: Elsevier-Saunders; 2006. pp 365-393.

14. Curci JJ, Horman MJ. Boerhaave's syndrome: the importance of early diagnosis and treatment. Ann Surg 1976;183: 401-8.

15. Phillips LGJr, Cunningham J. Oesophageal perforation. Radiol Clin North Am 1984;22:607-13.

16 Jones WG II, Ginsberg RJ. Oesophageal perforation: a continuing challenge. Ann Thorac Surg 1992; 53:534-43.

17. Suarez-Poveda T, Morales-Uribe $\mathrm{CH}$, Sanabria A, et al. Diagnostic performance of CT esophagography in patients with suspected esophageal rupture. Emerg Radiol 2014;21:505.

18. Endicott JN, Molony TB, Campbell G, et al. Oesophageal perforations: the role of computerized tomography in diagnosis and management decisions. Laryngoscope 1986;96:751-7.

19. Huber-Lang M, Henne-Bruns D, Schmitz B, et al. Esophageal perfora- 
tion: principles of diagnosis and surgical management. Surg Today 2006;36:332-40.

20. Walther B, Johansson J, Johnsson F, et al. Cervical or thoracic anastomosis after esophageal resection and gastric tube reconstruction: a prospective ran- domized trial comparing sutured neck anastomosis with stapled intrathoracic anastomosis. Ann Surg 2003;238:80314.

21. Talbot M, Yee G, Saxena P. Endoscopic modalities for upper gastrointestinal leaks, fistulae and perforations. Anz J
Surg 2017;87:171-17.

22. Sulpice L, Dileon S, Rayar M, et al. Conservative surgical management of Boerhaave's syndrome: experience of two tertiary referral centers. Intern J Surg 2013;11:64-7. 